\title{
EXTENSOR MECHANISM ALLOGRAFT IN TOTAL KNEE ARTHROPLASTY
}

\author{
Camilo Partezani Helito ${ }^{1}$, Riccardo Gomes Gobbl ${ }^{1}$, Mateus Ramos TozI ${ }^{1}$, Alessandro Monterroso Félix ${ }^{1}$, \\ Fábio Janson AngelinI ${ }^{1}$, José Ricardo Pécora ${ }^{1}$
}

\section{ABSTRACT}

Objective: To analyze the experience with allograft transplantation of the extensor mechanism in total knee arthroplasty and compare results with the international experience. Methods: We retrospectively evaluated three cases of extensor mechanism allograft after total knee arthroplasty performed in our hospital with the aid of one of the few tissue banks in Brazil and attempt to establish whether our experiences were similar to others reported in the world literature regarding patient indication, techniques, and outcomes. Results: Two cases went well with the adopted procedure, and one case showed bad results and progressed to amputation. As shown in the literature, the adequate tension of the graft, appropriate tibial fixation and especially the adequate patient selection are the better predictors of good outcomes. Previous chronic infection can be an unfavorable predictor. Conclusion: This surgical procedure has precise indication, albeit uncommon, either because of the rarity of the problem or because of the low availability of allografts, due to the scarcity of tissue banks in Brazil. Level of Evidence IV, Case Series.

Keywords: Knee. Arthroplasty. Allograft.

Citation: Helito CP, Gobbi RG, Tozi MR, Félix AM, Angelini FJ, Pécora JR. Extensor mechanism allograft in total knee arthroplasty. Acta Ortop Bras. [online]. 2013;21(6):315-9. Available from URL: http://www.scielo.br/aob.

\section{INTRODUCTION}

The rupture of the extensor mechanism is one of the most devastating complications after total knee arthroplasty (TKA). In this situation the prosthesis function becomes severely impaired, since the quadriceps is its main engine, besides compromising its stability. Compensatory mechanisms developed by patients to prevent involuntary flexion of the knee during gait invariably involve joint hyperextension (recurvatum), highly overloading the interface fixation of the prosthesis, leading to its losening. ${ }^{1-3}$

Fortunately this complication is rare. ${ }^{3,4}$ There are some risk factors that are involved in this situation, such as obesity, use of corticosteroids, hiperlaxity ligamentous, low patella, previous intervention in the extensor apparatus, prior patellectomy, patellofemoral malalignment, need for extensive patellar releases, manipulation by joint stiffness, revision arthroplasties and long term surgeries. ${ }^{5-7}$ From the anatomical point of view, the injury can happen: in the quadriceps tendon, patellar, secondary to patellar fracture or tibial tuberosity. However, it is common for fractures of the patella in TKA patients not to present with loss of the extensor function.

The treatment of rupture of the extensor apparatus depends on its anatomical location and viability of damaged structures. In acute cases where the injured structures are not degenerated the direct suture with or without tendon strengthening can bring good results.

The big challenges are chronic cases or degenerated tendons, often unidentifiable and therefore not capable of supporting the powerful traction of the quadriceps muscle even after attempts to suture. The primary suture, even with reinforcement usually leads to functionally unacceptable results. ${ }^{2-4,8-10}$

Under these conditions, the treatment with transplantation with the entire extensor mechanism allograft is becoming common. When respected technical details, this technique produces reasonable functional outcomes, although some may persist limitações. ${ }^{11-13}$

The experience of the Institute of Orthopedics and Traumatology, Hospital das Clínicas, Universidade de São Paulo with the transplant technique with homologous extensor mechanism in knee arthroplasties is desribed in this article.

\section{CASES AND METHODS}

A retrospective analysis of three patients with total knee arthroplasty underwent transplantation of the extensor mechanism

All the authors declare that there is no potential conflict of interest referring to this article.

1. Institute of Orthopedics and Traumatology, Hospital das Clínicas, Faculdade de Medicina, Universidade de São Paulo, São Paulo, SP, Brazil.

Work performed at LIM 41 - Laboratory of Medical investigation, Muscle skeleton Systgem, Department of Orthopedics and Traumatology, Faculdade de Medicina da Universidade de São Paulo, São Paulo, SP, Brasil.

Corresponding: Rua Dr. Ovídio Pires de Campos, 333, Cerqueira Cesar. 05403-010, São Paulo, SP, Brazil. camilo_helito@yahoo.com.br 
allograft at the Institute of Orthopedics and Traumatology, Hospital das Clinicas, Faculdade de Medicina, Universidade de São Paulo (IOT-HC/FMUSP). The grafts were obtained from the Tissue Bank, Institute of Orthopedics and Traumatology, Hospital das Clínicas, Faculdade de Medicina, Universidade de São Paulo, a pioneer in our country. The cases are described below: (Figure 1)

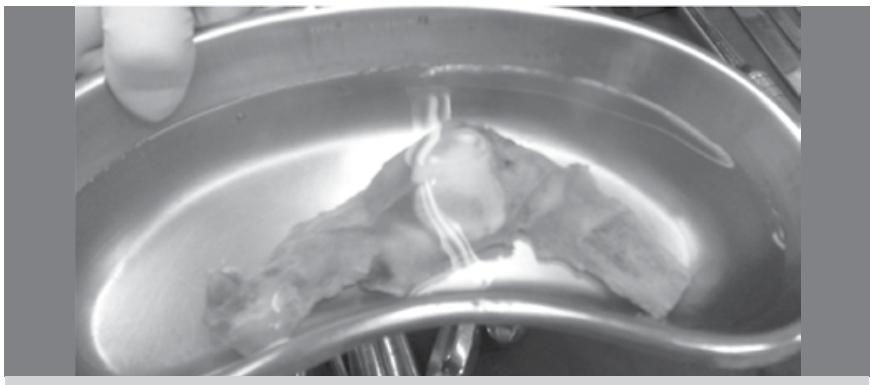

Figure 1. Extensor mechanism of the tissue bank, with the tibial insertion into the quadriceps tendon, including the patella.

Case 1

Male patient, 85 years old, with previous total left patellectomy due to patellofemoral arthritis at age 60 who subsequently underwent left TKA at age 79 ( surgeries performed in a different hospital ). He developed pain and progressive limitation of active and passive knee extension causing restriction of activities of daily living. He became accompanied by the Knee group from IOT-HC/FMUSP in December 2008, at that time four years and nine months after arthroplasty. On physical examination the patient had restriction on knee extension with lag of 40 degrees in active extension and 20 degrees in passive extension. The patient only walked with the aid of a walker, with no load on the compromised member. He did not show any ligament instability. Radiographs showed loosening of the tibial component with significant tear of the polyethylene insert. The inflammatory markers (ESR and CRP) were normal and bone scan was not suggestive of an infectious process. In April 2009 the patient underwent revision arthroplasty and allograft extensor mechanism transplant. The graft consisted of quadriceps tendon - patella (full) - patellar tendon - anterior tibial tuberosity (ATT). The graft was obtained from the IOT-HC/FMUSP Tissue Bank, having been previously prepared and frozen, without viable cartilage. The graft placement was based on the lower pole of the patella, which was kept $2.5 \mathrm{~cm}$ above the joint line. A pin in the tibia was inserted in the topography of the anterior tuberosity, where the previously molded plug bone allograft (ATT) was fitted under pressure and fixed with a large fragments screw. The articular surface of the patella graft was not replaced for being insensitive; replacement could be an unnecessary source of complications (such as fracture). The quadriceps tendon graft was then sutured to the patient's quadriceps, tensing toward the graft proximal and distal quadriceps, through Kracow technique with high strength nonabsorbable multifilament wire, keeping the knee in full extension. The capsule and what remained of the remnant extensor apparatus were sutured over the graft with simple absorbable wire, covering it in an attempt to increase its vascularization. (Figures 2 and 3) Postoperatively the patient was maintained immobilized in extension for six weeks performing only isometric strengthening exercises and assisted passive range of motion gain. After the sixth week active exercises with progressive weight were allowed until the third month, when immobilization was discontinued.

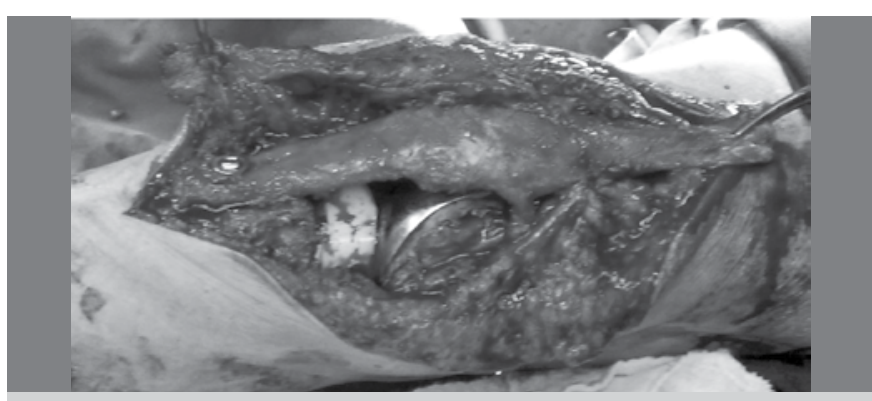

Figure 2. Allograft implanted in the tibia and fixed with screws.
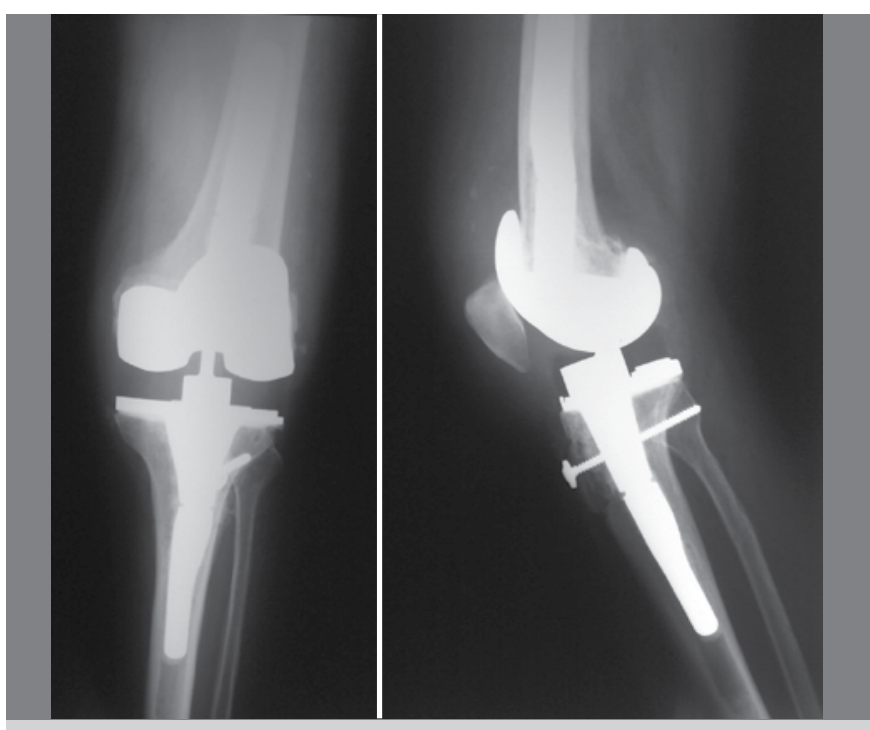

Figure 3. Final radiographic appearance after revision with extensor mechanism transplant.

Case 2

Female oriental 80 years old patient underwent primary total knee arthroplasty for osteoarthritis in 1997 by another hospital. It initially evolved well, but later presented infection leading to septic loosening of the prosthesis, which was diagnosed in 2006. In 2007 she started being followed-up in our hospital. She presented, at this time, range of motion of $10-80^{\circ}$.

As the patient presented symptoms of chronic infection associated with loosening of the components, treatment with review in two-steps was chosen according to our hospital protocol. ${ }^{14,15}$ The patient underwent removal of the prosthesis, thorough debridement and spacer with cement associated with vancomycin. During the procedure, it was necessary to resect a large part of the patellar tendon that was found damaged by the infection, the extensor mechanism becoming consequently compromised. Throughout her treatment, she was also accompanied by the hospital's infection service that indicated antibiotic therapy. The cultures the first cleaning showed no bacterial growth and the infection was controlled, with normalization of inflammatory 
activity (ESR and CRP). Thus, the patient was submitted two months after the second time with revision arthroplasty and transplantation of the extensor mechanism allograft.

The graft consisted of quadriceps tendon-patella (full) - patellar tendon-anterior tibial tuberosity (ATT). The graft was obtained from the Tissue Bank of IOT-HC/FMUSP, having been previously prepared and frozen, without viable cartilage. The technical details of the surgery and the initial postoperative were similar to those of case 1. We point out the differences between them: the bone plug fixation was performed with ATT flexible steel wires and we chose to perform patellar allograft replacement by a technique identical to that of a patellar component in a primary arthroplasty.

Case 3

Female white 81 years old retired patient. She underwent total right knee arthroplasty in 2007. On the $4^{\text {th }}$ postoperative day, when leaving the hospital after being discharged, she felt to the ground with trauma in the anterior knee that caused wound dehiscence and exposure of the prosthesis with loss of patellar component. The patient was again hospitalized and underwent aggressive surgical procedure for cleaning and collection of cultures and antibiotic therapy for six weeks to control a local infectious process, multi - sensitive Staphylococcus aureus having been detected. Intraoperatively, resection of $60 \%$ of the patella was required due to its fragmentation. The patient recovered well from the clinical point of view, and local infection. Progressively during the follow-up, she started to present pain and disability of knee extension due to lateral dislocation of the extensor mechanism to active quadriceps contraction due to the lack of restraint on the local soft parts after surgical debridement. Due to the lateralization of the extensor mechanism, she slowly progressed to medial instability and large valgus opening between 2007 and 2011. The patient did not present any evidence of loosening of the femoral and tibial components of the arthroplasty. The functional limitation became important, and surgical treatment was the option elected.

In October 2011 she underwent reconstruction of the extensor mechanism and the medial collateral ligament with homologous graft from a tissue bank using a complete extensor mechanism (quadriceps tendon - patella - patellar tendon - tibial tuberosity) and two flexor tendons to medial ligament complex. A channel in region of the anterior tibial tuberosity was performed to accommodate a "press fit" plug bone graft associated with 4.5- $\mathrm{mm}$ cortical screw with washer.

Intraoperatively it was observed that the tibial and femoral components were fixed and well positioned and exchange was not performed.

Patient developed an active knee extension at the immediate postoperative period. The post-operative procedures were also similar to those of cases 1 and 2 .

\section{RESULTS}

\section{Case 1}

We consider this result a success.

He did not present in the initial postoperative period any infectious or healing complications.

After twelve month follow-up the patient presents no pain, no instability, walking with the aid of a cane, with full passive extension and partial active extension (lag of 10 degrees) and flexion of 90 degrees. He presents quadriceps strength grade IV (strengths against some resistance).

The patient is very satisfied with the result. (Figure 4)

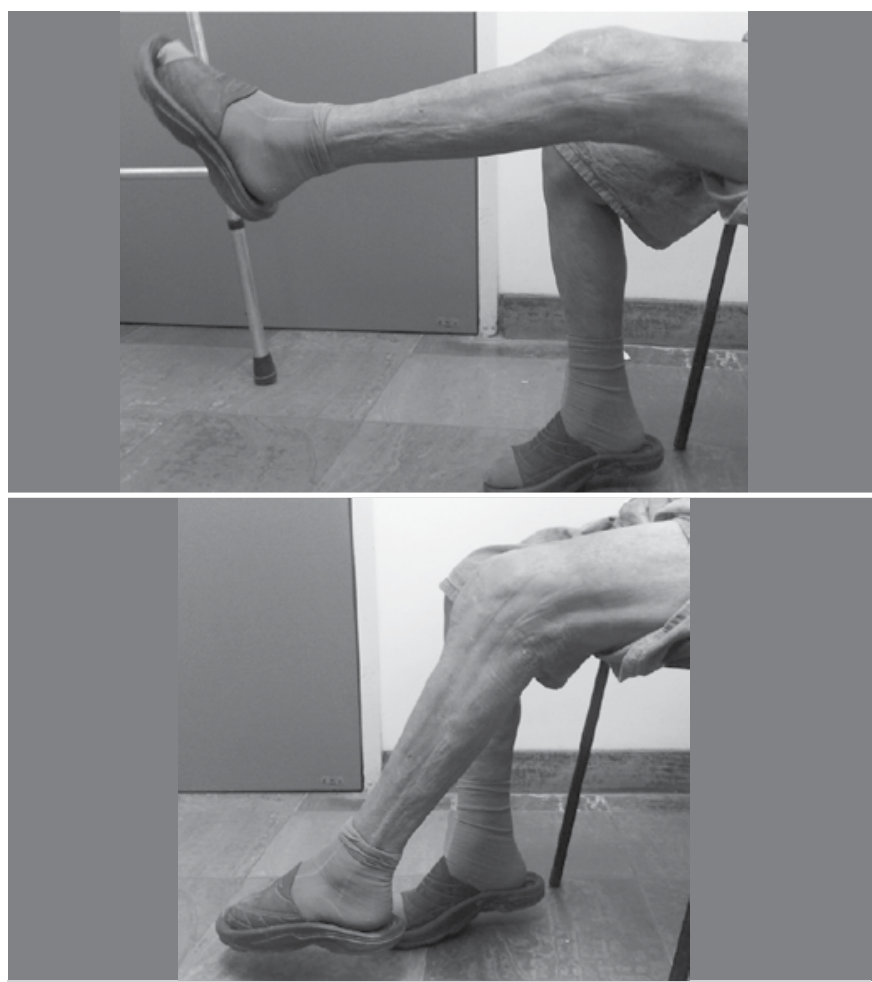

Figure 4. Early postoperative period, showing active extension and flexion obtained while in the process of physiotherapy gain.

Case 2

This patient developed differently from case 1 .

One month postoperatively, the patient had a satisfactory outcome of the functional point of view. She presented on this occasion passive and active arc of motion of $0-70^{\circ}$ and $20-40^{\circ}$, respectively, reaching $20-90^{\circ}$ for the active arc of motion on the $2^{\text {nd }}$ postoperative month.

However, it soon evolved to the formation of a pre-patellar abscess. It was then submitted to a new surgical cleaning, this time without removal of the prosthesis since it was an acute infection, with the possibility of preservation of the implant. In this surgery infectious agents have been identified as $E$. faecalis and Pseudomonas aeruginosa. She received appropriate antibiotics, but since no satisfactory control of infection has been achieved, the patient underwent three more surgical dressings (including the removal of flexible steel wire) within two months. Since a secreting fistula was still present after these procedures, we decided to remove the prosthesis and the allograft, and a new spacer with antibiotics was implanted. (Figure 5)

Despite the removal of the implants, the case did not progress well, with wound dehiscence and resistance of the infection despite the use of a VAC system (vacuum-assisted closure system) and underwent four surgical dressings within a month. Due to the severity of the infection and in agreement with the 
patient and her family, we chose the transfemoral amputation as definitive treatment since there was no prospect of placing new prosthesis.

After the amputation, it progressed well, with control of the infection and the patient is currently being followed up at our hospital by the Prosthetics/Amputees group.

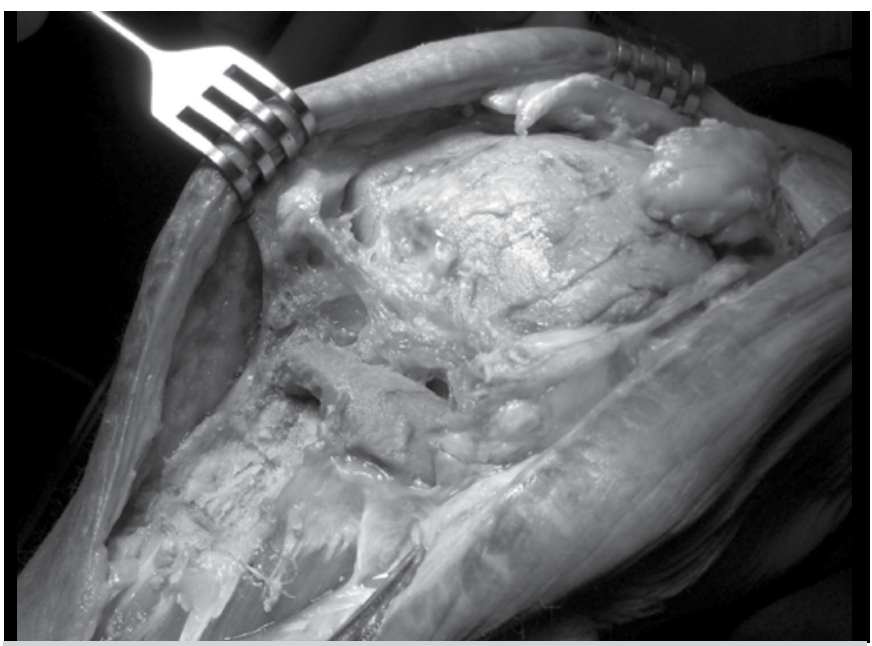

Figure 5. Appearance of case 2 after removal of the prosthesis and allograft without extensor mechanism.

\section{Case 3}

The patient was doing satisfactorily until the fourth postoperative month when she developed pain and difficulty walking. Radiography revealed a peri-prosthetic fracture of the tibial component in the metaphyseal region below the tip of the tibial component, however, with an early callus formation. The patient was clinically well, being able to walk with a walker. Probably the fracture occurred in an inert way, and at diagnosis it has already progressed to consolidation. Thus, we chose a conservative treatment with an inguinal malleolus knee immobilizer and load restriction. After two months of treatment, patient had active knee extension, quadriceps strength grade IV and arc of motion of 0-100 degrees painlessly.

\section{DISCUSSION}

Some case series have been published with reasonable results of allograft extensor mechanism. Emerson et al.${ }^{16}$ did not obtain extension lag in six of nine patients with an 4.1 years of follow-up on average after using this technique (two patients had lag $<25^{\circ}$ and one of $40^{\circ}$ ). Five patients walked unaided, three used canes and one used a walker. Complications that have been described include one case of graft rupture, a failure of the suture with the quadriceps, a loosening of the patellar component and a patella fracture after revision component. The authors currently consider that the replacement of the articular surface of the patella should be avoided. Another series published by Nazarian and Booth ${ }^{17}$ presented results of 36 patients who underwent this technique with an average of 3.6 years of follow up, of which 21 presented total active extension (without lag). In 15 patients, the average lag was $13^{\circ}$. There were eight cases of failure of the allograft, which have been treated with a new allograft. Six of them failed at the suture of the quadriceps and two in ATT. Nevertheless, the result was considered successful in 34 patients. However, there are series with poor results, such as the one published by Leopold et al. ${ }^{18}$ In seven patients undergoing allograft all remained with extender lag of $30^{\circ}$ or more, although the authors considered that the lack of proper tension at the time of the surgery may have been a cause of failure. Only one patient did not use any assistance to walk in full time.

Despite these poor results, international experience suggests that this technique is one of the best options for ruptures of the extensor mechanism in patients with total knee arthroplasty, even in acute cases. Technical recommendations for the success of the surgery are mainly: release the quadriceps and native tendon from recesses; strongly tension the graft in extension (loosening always occurs post- op), the bone plug of ATT should be inserted into the trough created by the surgeon by press-fit and be immobilized with steel wires or screws; immobilization should be continuous in length for six weeks and the use of protective brace should be done within three months, the height of the lower pole of the patella should be around $1 \mathrm{~cm}$ above the interlining (although in our study we have succeeded using $2.5 \mathrm{~cm}$ ) and proper selection of pacientes. ${ }^{13}$ Contraindications to this surgical procedure are cases of active infection and patients who cannot keep the immobilization required postoperatively or perform the appropriate rehabilitation program. For the group to which the reconstruction of the extensor mechanism is not indicated, there remains the possibility of arthrodesis or use of orthoses. ${ }^{5}$

In our case series we had two good results (although one of the cases had only eight months follow-up) and one bad outcome. We recall that an important factor for the success of the procedure is the selection of the patient. This procedure takes into account both the technical difficulty to perform the surgery (cases that concomitantly undergo a highly complex revision arthroplasty tend to produce worse results than a transplantation of the extensor mechanism by simple rupture) as well as the patient's own factors (such as vascular disorders, rheumatic diseases, etc.) which independently increase the risk of postoperative complications.

It is interesting to note that the case with poor outcome presented early infection despite it was apparently controlled. By inserting an inert (avascular) tissue in the knee, we currently indicate transplantation in cases of aseptic revision, despite not considering prior infection an absolute contraindication if it is well treated, since the third case showed acute infection that was treated properly and the reconstruction was successful until now.

The other differences in our cases were basically the method of attachment of ATT (screws in two cases and ligature steel wire in the other) and the replacement of the articular surface of the patella. Regarding the fixation method, we prefer to use screws, but its use is not always possible because there is an implant rod within the spinal canal, which limits its placement. On patellar replacement, we currently do not perform it any more due to the risk of fractures (case 2, despite having been described later, was performed chronologically before). We cannot tell if patellar replacement contributes to the occurrence of infection, but probably the biggest risk is related to the weakening of the extensor mechanism. 
Currently in our hospital the preferred procedure in cases of patellar fracture in patients with total knee arthroplasty is still nonsurgical treatment with immobilization for about six weeks and functional rehabilitation, since the extensor mechanism is preserved. In cases where there is full compromise of the extensor mechanism, either by fracture or rupture of the patellar tendon, we decided directly by transplantation of the extensor mechanism, since our experience and the literature show highly unsatisfactory results with the repair attempt, reaching failures in over $90 \%$ of casos. ${ }^{19}$

Transplantation of the extensor apparatus is currently considered one of the best solutions for this rare complication (rupture of the extensor mechanism), being widely indicated outside
Brazil. In our environment, on the other hand, experience with this technique has not been reported.

\section{CONCLUSION}

Our cases showed two satisfactory results and one poor result, probably due to inadequate patient choice. Nevertheless, we consider the surgery a procedure of precise indication, however unusual, either due to the rarity of the problem or due to the low availability of allografts by the scarcity of tissue banks in the country. Anyway, this technique has proven useful and with good results (in well-selected patients) in cases of total knee arthroplasty complicated by rupture of the extensor mechanism.

\section{REFERENCES}

1. MacCollum MS, Karpman RR. Complications of the PCA anatomic patella. Orthopedics. 1989;2(11):1423-8.

2. Doolittle $\mathrm{KH}$ 2nd, Turner $\mathrm{RH}$. Patellofemoral problems following total knee arthroplasty. Orthop Rev. 1988;17(7):696-702.

3. Rand JA, Morrey BF, Bryan RS. Patellar tendon rupture after total knee arthroplasty. Clin Orthop Relat Res. 1989;(244):233-8.

4. Lynch AF, Rorabeck $\mathrm{CH}$, Bourne RB. Extensor mechanism complications following total knee arthroplasty. J Arthroplasty. 1987;2(2):135-40.

5. Springer BD, Della Valle CJ. Extensor mechanism allograft reconstruction after total knee arthroplasty. J Arthroplasty. 2008;23(Suppl 7):35-8.

6. Rand JA. Extensor mechanism complications following total knee arthroplasty. J Knee Surg. 2003;16(4):224-8.

7. Kulkarni M, Sawant M, Ireland J. Allograft reconstruction of the extensor mechanism for progressive extensor lag after total knee arthroplasty and previous patellectomy - 3-Year Follow-Up. J Arthroplasty. 1999;14(7):892-4.

8. Malhotra R, Garg B, Logani V, Bhan S. Management of extensor mechanism deficit as a consequence of patellar tendon loss in total knee arthroplasty: a new surgical technique. J Arthroplasty. 2008;23(8):1146-51.

9. Abril JC, Alvarez L, Vallejo JC. Patellar tendon avulsion after total knee arthroplasty. A new technique. J Arthroplasty. 1995;10(3):275-9.

10. Wilson FC, Venters GC. Results of knee replacement with the Walldius prosthesis: an interim report. Clin Orthop Relat Res. 1976;(120):39-46.

11. Nazarian DG, Booth RE Jr. Extensor mechanism allografts in total knee arthro- plasty. Clin Orthop Relat Res. 1999;(367):123-9.

12. Burnett RS, Berger RA, Della Valle CJ, Sporer SM, Jacobs JJ, Paprosky WG, et al. Extensor mechanism allograft reconstruction after total knee arthroplasty. J Bone Joint Surg Am. 2005;87(Suppl 1 Pt 2):175-94.

13. Burnett RS, Berger RA, Paprosky WG, Della Valle CJ, Jacobs JJ, Rosenberg AG. Extensor mechanism allograft reconstruction after total knee arthroplasty. A comparison of two techniques. J Bone Joint Surg Am. 2004;86(12):2694-9.

14. D’Elia CO, dos Santos AL, Leonhardt MC, Lima AL, Pécora JR, Camanho GL. Tratamento das infecções pós artroplastia total de joelho: resultados com 2 anos de seguimento. Acta Ortop Bras. 2007;15(3):158-62.

15. Lima AL, Pécora JR, Albuquerque RM, de Paula AP, D’Elia CO, dos Santos $\mathrm{AL}$, et al. Infecção pós-artroplastia total do joelho - Considerações e protocolo de tratamento. Acta Ortop Bras. 2004;12(4):236-41.

16. Emerson RH Jr, Head WC, Malinin TI. Extensor mechanism reconstruction with an allograft after total knee arthroplasty. Clin Orthop Relat Res. 1994;(303):79-85.

17. Nazarian DG, Booth RE Jr. Extensor mechanism allografts in total knee arthroplasty. Clin Orthop Relat Res. 1999;(367):123-9.

18. Leopold SS, Greidanus N, Paprosky WG, Berger RA, Rosenberg AG. High rate of failure of allograft reconstruction of the extensor mechanism after total knee arthroplasty. J Bone Joint Surg Am. 1999;81(11):1574-9.

19. Rand JA, Morrey BJ, Bryan RS. Patellar tendon rupture after total knee arthroplasty. Clin Orthop Relat Res. 1989;(244):233-9. 